\title{
Lowness for Difference Tests
}

\author{
David Diamondstone and Johanna N. Y. Franklin
}

\begin{abstract}
We show that being low for difference tests is the same as being computable and therefore lowness for difference tests is not the same as lowness for difference randomness. This is the first known example of a randomness notion where lowness for the randomness notion and lowness for the test notion do not coincide. Additionally, we show that for every incomputable set $A$, there is a difference test $\mathcal{T}^{A}$ relative to $A$ which cannot even be covered by finitely many unrelativized difference tests.
\end{abstract}

Along with the study of random sequences comes the study of sequences that are far from random. There are many different ways in which an infinite binary sequence can be said to be far from random, such as triviality, lowness, lowness for tests, or being a base (see Downey and Hirschfeldt [3], Nies [10]). In this paper we will focus on the lowness notions.

The concept of lowness has been studied in many contexts. In general, a sequence is said to be low if it is indistinguishable from a computable sequence in the given context. For instance, a sequence $A$ is low with respect to the Turing jump if $A^{\prime} \equiv_{T} 0^{\prime}$, that is, if its jump has the same Turing degree as that of a computable sequence. Lowness has also been studied in the contexts of learning theory (see Slaman and Solovay [11]) and recursive model theory (see Franklin and Solomon [7]), but it is most prominent in algorithmic randomness (see [5]).

The first notion is that of lowness for $\mathcal{R}$, and the second is that of lowness for tests.

Definition 1 A sequence $A$ is low for a randomness notion $\mathcal{R}$ if $\mathcal{R}^{A}=\mathcal{R}$, that is, if it does not make any random sequence appear to be nonrandom when it is used as an oracle.

Definition 2 Let $\mathcal{R}$ be a randomness notion. A sequence $A$ is low for $\mathcal{R}$-tests if for every $\mathcal{R}$-test relative to $A\left\langle V_{i}^{A}\right\rangle$ there is an unrelativized $\mathcal{R}$-test $\left\langle U_{i}\right\rangle$ such that $\bigcap_{i} V_{i}^{A} \subseteq \bigcap_{i} U_{i}$.

Received May 10, 2012; accepted June 26, 2012

2010 Mathematics Subject Classification: Primary 03D32

Keywords: algorithmic randomness, difference randomness, difference tests, lowness

(C) 2014 by University of Notre Dame 10.1215/00294527-2377878 
It is clear that for any notion $\mathcal{R}$, any sequence that is low for $\mathcal{R}$-tests must also be low for $\mathcal{R}$, but the converse is not obviously true since more than one unrelativized test may be required to cover a relativized test. Whenever a universal test exists, as for Martin-Löf randomness, these notions are clearly equivalent. If this is not the case, some work must be done to determine whether this equivalence holds. For instance, there is no universal Schnorr test. However, Kjos-Hanssen, Nies, and Stephan [8] showed that every sequence that is low for Schnorr randomness is low for Schnorr tests by characterizing them as the recursively traceable sets, the class which Terwijn and Zambella [13] had shown were precisely the sets that were low for Schnorr randomness.

Bienvenu and Miller [1] have recently characterized lowness in terms of open covers in a way that suggests that lowness for tests and lowness will coincide for any randomness notion where the tests are composed of $\Sigma_{1}^{0}$-sets. In this paper, we consider difference randomness, in which the test elements are differences of $\Sigma_{1}^{0}$-sets, and show that lowness for difference tests does not coincide with lowness for difference randomness. This is the first example of a randomness notion for which these classes are known to be different and suggests that there is no intrinsic reason for these classes to coincide. One could make the argument that difference randomness is not a natural randomness notion and that this example is therefore pathological; however, the difference random sets are precisely the Turing incomplete Martin-Löf random sets (see Franklin and $\mathrm{Ng}$ [6]) and as such form a very natural subclass of the Martin-Löf random sets (see Stephan [12]).

\section{Introduction}

In Section 2, we prove our main theorem.

Theorem 3 If $A$ is low for difference tests, then $A$ is computable.

Then, in Section 3, we show that incomputable sets are very far from being low for difference randomness.

Theorem 4 If $A$ is incomputable, then there is a difference test $\mathcal{T}^{A}$ relative to $A$ which cannot be captured by finitely many unrelativized difference tests.

Our notation is standard and will generally follow [10]. We will not define difference randomness as Franklin and $\mathrm{Ng}$ [6] originally did, but the following formulation is easily seen to be equivalent and is more appropriate for our purposes.

Definition 5 A difference test is a pair $\left(\left\langle U_{n}\right\rangle, \mathcal{C}\right)$, where $\left\langle U_{n}\right\rangle$ is a uniformly $\Sigma_{1}^{0}$-sequence and $\mathcal{C}$ is a $\Pi_{1}^{0}$-class such that for all $n$, the measure condition

$$
\mu\left(u_{n} \cap \varphi\right)<2^{-n}
$$

is satisfied.

We say that a difference test captures a real $X$ if $X \in \bigcap_{n} u_{n} \cap \mathcal{C}$. A real $X$ is called difference random if it is not captured by any difference test.

Definition $6 \quad$ A real $X$ is low for difference randomness if every real which is difference random is difference random relative to $X$. A real $X$ is low for difference tests if for every difference test $\left(\left\langle\mathcal{U}_{n}^{X}\right\rangle, \mathcal{C}^{X}\right)$ relative to $X$, there is an unrelativized difference test $\left(\left\langle\mathcal{V}_{n}\right\rangle, \mathcal{D}\right)$ such that

$$
\bigcap_{n} u_{n}^{X} \cap \mathcal{C}^{X} \subseteq \bigcap_{n} \mathcal{V}_{n} \cap \mathcal{D}
$$


If equation (1) holds, then we say that $\mathcal{T}^{X}=\left(\left\langle\mathcal{U}_{n}^{X}\right\rangle, \mathcal{C}^{X}\right)$ is covered by $\mathcal{T}^{\prime}=\left(\left\langle\mathcal{V}_{n}\right\rangle\right.$, $D)$. This can be generalized to finitely many tests $\mathcal{T}_{1} \cdots \mathcal{T}_{n}$ in the obvious way.

All of the proofs in this paper are done in the space [0,1], but all of the results hold identically for $2^{\omega}$ as there is a computable measure isomorphism $X \mapsto 0 . X$ from $2^{\omega}$ to $[0,1]$.

\section{Lowness for Difference Tests}

To show that no incomputable set is low for difference randomness, we begin with hyperimmune sets, that is, those sets that compute a function that cannot be dominated by any computable function. We show how to use such a function $f$ to build an $f$-difference test $\mathcal{T}^{f}$ which is not covered by any single unrelativized difference test $\mathcal{T}^{\prime}$. The first part of our strategy is to ensure that $\mathcal{T}^{f}$ captures $\Omega$, which ensures that $\mathcal{T}^{f}$ is not covered by any difference test $\mathcal{T}^{\prime}$ which does not capture $\Omega$; we then need only concern ourselves with difference tests $\mathcal{T}^{\prime}$ which capture $\Omega$. Since $\Omega$ is Martin-Löf random, a difference test $\mathcal{T}^{\prime}$ which captures $\Omega$ cannot be a Martin-Löf test. Moreover, this must hold locally in any interval around $\Omega$. Such a $\mathcal{T}^{\prime}$ must be missing many open intervals near $\Omega$, which will be revealed as they show up in the $\Pi_{1}^{0}$-class that makes $\mathcal{T}^{\prime}$ a difference test rather than a Martin-Löf test. When we see such an interval, we can ensure that some rational in the interval is captured by $\mathcal{T}^{f}$. We use places where $f$ escapes domination to ensure that we see such an interval soon enough that we can use it to diagonalize, that is, before we have put it into the complement of the $\Pi_{1}^{0}[f]$-class that we are building as part of the test $\mathcal{T}^{f}$.

Theorem $7 \quad$ Let $A$ have hyperimmune degree. Then $A$ is not low for difference tests.

Proof Let $f \leq_{T} A$ escape domination by every computable function $g$. We will use $f$ to define a difference test $\left(\left\langle u_{n}^{A}\right\rangle, \complement^{A}\right)$. Let $\left\langle\Omega_{s}\right\rangle$ be a monotone approximation of the halting probability $\Omega$. We define the difference test by setting

$$
u_{n}^{A}=\left[0, \Omega+2^{-n}\right)
$$

and constructing $\mathcal{C}^{A}$ in stages. Let $\left\langle D_{e}\right\rangle$ be an effective enumeration of all $\Pi_{1}^{0}$ classes, where $\mathscr{D}_{e}[s]$ is the stage $s$ approximation to $\mathscr{D}_{e}$. During the construction, we will enumerate a set of rationals $Q$; when a rational is enumerated into $Q$, it will be enumerated for some $\mathscr{D}_{e}$, and the requirement $\mathcal{R}_{e}$ will henceforth be marked as satisfied.

2.1 Construction Stage 0 : Let $\mathcal{C}^{A}[0]=[0,1]$, and let $Q_{0}=\emptyset$.

Stage $s$ : For each $e<s$ with $\mathcal{R}_{e}$ unsatisfied, search for the least rational $q$ such that

(1) $\Omega_{s-1}<q<\Omega_{f(s)}$,

(2) $q \notin \mathscr{D}_{e}[f(s)]$, and

(3) the denominator of $q$ is at most $f(s)$.

For each $e$ such that some $q$ is found, enumerate that $q$ into $Q$, and mark $\mathcal{R}_{e}$ as satisfied. Then remove the set $\left[0, \Omega_{s}\right) \backslash Q$ (which is a finite union of open ${ }^{1}$ intervals) from $C^{A}$. 


\subsection{Verification}

Lemma 8 We have $\mathcal{C}^{A}=Q \cup[\Omega, 1]$.

Proof We will show, by induction on $s$, that for all $s$,

$$
\bigodot^{A}[s] \supseteq Q_{s} \cup\left[\Omega_{s}, 1\right] .
$$

At stage $0, \bigodot^{A}[0]=[0,1]$, so this is certainly true. Assume that

$$
\bigodot^{A}[s-1] \supseteq Q_{s-1} \cup\left[\Omega_{s-1}, 1\right] .
$$

If any rational numbers are enumerated into $Q$ at stage $s$, they must be at least $\Omega_{s-1}$, so

$$
\bigodot^{A}[s-1] \supseteq Q_{s} \cup\left[\Omega_{s}, 1\right] .
$$

At stage $s$, no element of $Q_{s}$ is removed from $\bigodot^{A}$, nor is anything larger than $\Omega_{s}$ removed, and therefore

$$
\bigodot^{A}[s] \supseteq Q_{s} \cup\left[\Omega_{s}, 1\right] .
$$

By induction, this holds for all $s$. Taking the lim inf on both sides, we have

$$
\varphi^{A}=\liminf _{s} \varphi^{A}[s] \supseteq \liminf _{s} Q_{s} \cup\left[\Omega_{s}, 1\right] .
$$

Thus

$$
\bigodot^{A} \supseteq Q \cup[\Omega, 1]
$$

On the other hand, if $x<\Omega$ and $x \notin Q$, then there is some $s$ such that $x<\Omega_{s}$. Then $x$ is removed from $\mathcal{C}^{A}$ by the end of stage $s$. Thus

$$
\varphi^{A}=Q \cup[\Omega, 1]
$$

as claimed.

From this, we can easily see that the measure condition is satisfied, that is, $\left(\left\langle u_{n}^{A}\right\rangle, \bigodot^{A}\right)$ is a difference test, and that

$$
\bigcap_{n} u_{n}^{A} \cap \mathcal{C}^{A}=[0, \Omega] \cap(Q \cup[\Omega, 1])=Q \cup\{\Omega\} .
$$

Lemma 9 There is no difference test such that its intersection contains

$$
\bigcap_{n} u_{n}^{A} \cap \mathcal{C}^{A}=Q \cup\{\Omega\}
$$

Proof Consider a difference test $\left(\left\langle\mathcal{V}_{n}\right\rangle, \mathscr{D}\right)$. There are two cases: when this difference test captures $\Omega$, and when it does not. It the test fails to capture $\Omega$, then certainly the intersection cannot contain $Q \cup\{\Omega\}$. Suppose that the difference test does capture $\Omega$. Then for every $s$, the interval $\left(\Omega_{s}, \Omega\right)$ cannot be a subset of $\mathcal{D}$, or else $\left\langle\left(\Omega_{s}, \Omega+2^{-n-1}\right) \cap \mathcal{V}_{n+1}\right\rangle$ would satisfy the Martin-Löf measure condition and would therefore be a Martin-Löf test capturing $\Omega$.

Let $e$ be an index of $D$. If $\mathcal{R}_{e}$ is ever marked as satisfied, there is some $q \notin \mathscr{D}_{e}$ which is enumerated into $Q$. By the above lemma, $q \in \bigcap_{n} U_{n}^{A} \cap \mathcal{C}^{A}$ but $q \notin \bigcap_{n} \mathcal{V}_{n} \cap \mathcal{D}$, so $q$ witnesses that the difference test $\left(\left\langle U_{n}^{A}\right\rangle, \mathcal{C}^{A}\right)$ is not covered by the difference test $\left(\left\langle\mathcal{V}_{n}\right\rangle, \mathcal{D}\right)$. So it suffices to show that $\mathcal{R}_{e}$ is eventually marked satisfied.

In any interval of the form $\left(\Omega_{s-1}, \Omega\right)$, there must be some rational subinterval removed from $\mathscr{D}=\mathscr{D}_{e}$. We will define a computable function $g: \omega \rightarrow \omega$. To define $g(s)$, search for some natural number $n$ and rational number $q$ with denominator 
at most $n$ such that $\Omega_{s-1}<q<\Omega_{n}$ and some interval containing $q$ is removed from $\mathscr{D}_{e}$ by stage $n$; let $g(s)$ be the least such $n$. The above argument shows that such an $n$ will always be found, and so $g$ is a total computable function. Since $f$ escapes domination by $g$, there is a stage $s>e$ with $f(s)>g(s)$. During stage $s$ of the construction, the search for a rational number $q$ meeting conditions (1)-(3) will succeed, so $\mathcal{R}_{e}$ will be marked as satisfied during stage $s$ if it is not already so marked.

Therefore, $A$ is not low for difference tests. Theorem 7 is proved.

Corollary 10 If $A$ is low for difference tests, then $A$ is computable.

Proof If $A$ is $\Delta_{2}^{0}$ and incomputable, then $A$ is hyperimmune, whence $A$ is not low for difference tests.

Downey, Nies, Weber, and $\mathrm{Yu}$ [4] showed that the $K$-trivial sets are the sets that are low for weak 2-randomness versus Martin-Löf randomness, and since difference randomness is intermediate between these notions (see [6]), we know that every set that is low for difference randomness is $K$-trivial.

If $A$ is not $\Delta_{2}^{0}$, then $A$ is not $K$-trivial and hence not low for difference randomness. A fortiori, $A$ is not low for difference tests.

In fact, the sets which are low for difference randomness are precisely the $K$-trivials. Franklin and $\mathrm{Ng}$ [6] observed that for computably enumerable (c.e.) sets $A$, lowness for difference randomness is equivalent to another property, being weakly $M L$ noncuppable, meaning that there is no Martin-Löf random set $B \Varangle_{T} \quad \emptyset^{\prime}$ such that $\emptyset^{\prime} \leq_{T} A \oplus B$. Weak ML-noncuppability was recently shown by Day and Miller [2] to be the same as $K$-triviality. Since every $K$-trivial set is below a c.e. $K$-trivial (see Nies [9]), every $K$-trivial set is low for difference randomness.

This equivalence gives us the following additional corollary.

Corollary 11 Lowness for difference tests and lowness for difference randomness do not coincide.

Remark 12 It is worth pointing out that in order to satisfy requirement $\mathcal{R}_{e}$ diagonalizing against the $e$ th difference test - the construction completely ignores the sequence of $\Sigma_{1}^{0}$-classes which forms the first half of the test and focuses entirely on the $\Pi_{1}^{0}$-class $\mathscr{D}_{e}$ which forms the second half of the test. As such, it does not matter whether we diagonalize against an enumeration of all difference tests or merely an enumeration of all $\Pi_{1}^{0}$-classes.

The verification, however, does depend on the entire difference test, because whether we succeed at diagonalizing against a difference test using some element of $Q$ or using $\Omega$ depends on whether the difference test captures $\Omega$.

\section{How Far from Low?}

Theorem 3 tells us that no incomputable set is low for difference tests but does so in a very weak way. Examining the test $\mathcal{T}^{A}$ built by the proof, one sees that apart from $\Omega$, every real captured by $\mathcal{T}^{A}$ is rational. Hence each real captured by $\mathcal{T}^{A}$ is either captured by a single difference test capturing $\Omega$ or by a universal Martin-Löf test. One is therefore led to ask whether this is an essential feature of the proof. We know that incomputable $K$-trivials are low for difference randomness but not difference tests, but might they be somehow very close to being low for difference tests? In this 
section we show that this is not the case and that in fact every incomputable set is as far as possible from being low for difference tests in the following way.

Theorem 13 If $A$ is incomputable, there is a difference test $\mathcal{T}^{A}$ relative to $A$ which cannot be covered by finitely many unrelativized difference tests.

To prove this theorem, we can again focus on sets of hyperimmune degree, since incomputable sets not of hyperimmune degree cannot even be low for difference randomness. We use a variation on our strategy from Section 2. Again we build a test $\mathcal{T}^{f}$ and ensure that it captures $\Omega$, but now we have to diagonalize against finite unions of difference tests of the form $\mathcal{T}_{1} \cdots \mathcal{T}_{m}$. Again, in order to capture $\mathcal{T}^{f}$ at least one of these tests, say, $\mathcal{T}_{i}$, must contain $\Omega$. We can use the same strategy from before to find some interval $I$ in the complement of $\mathcal{T}_{i}$. We then repeat the same strategy inside $I$ but now only have to worry about $m-1$ of the tests from the union. Continuing like this for up to $m$ steps if necessary, we diagonalize against the entire ensemble $\mathcal{T}_{1} \cdots \mathcal{T}_{m}$. To prevent strategies for separate requirements from interfering with each other, we will work with infinitely many disjoint open subintervals of $[0,1]$,

$$
\left\{I_{E}: E \subset \omega \text { is finite }\right\} .
$$

(We could have done this in the previous construction as well, but it was unnecessary there.)

Theorem $14 \quad$ Let A have hyperimmune degree. Then there is a difference test relative to $A$ which cannot be covered by any finite collection of unrelativized difference tests.

Proof As before, we will build an $A$-difference test $\mathcal{T}^{A}=\left(\left\langle U_{n}^{A}\right\rangle, \mathcal{C}^{A}\right)$, defined using $f \leq_{T} A$, which escapes every computable function. Let $\left\langle\mathcal{T}_{e}\right\rangle$ be an enumeration of all unrelativized difference tests, where $\mathcal{T}_{e}=\left(\left\langle V_{n}^{e}\right\rangle, \mathscr{D}_{e}\right)$. We need to satisfy the requirements

$$
\mathcal{R}_{E}: \mathcal{T}^{A} \text { is not covered by }\left\{\mathcal{T}_{e}: e \in E\right\},
$$

where $E$ ranges over all finite subsets of the natural numbers. We will work for requirement $\mathcal{R}_{E}$ on the interval $I_{E}$, so in our construction we may fix a specific requirement and define $\left\langle U_{n}^{A}\right\rangle$ and $\mathcal{C}^{A}$ restricted to that interval for that requirement, ignoring all other requirements. To ensure that we can put all the separate pieces together and get a valid difference test, we need only ensure that

$$
\mu\left(U_{n}^{A} \cap \mathcal{C}^{A} \cap I_{E}\right) \leq \epsilon_{E} 2^{-n},
$$

where $\epsilon_{E}$ is a computable real-valued function of $E$ (a finite set of natural numbers) with $\sum_{E} \epsilon_{E}=1$.

Once again, we fix a monotone approximation $\left\langle\Omega_{s}\right\rangle$ of $\Omega$. For an interval $I$ with rational endpoints $a, b$, let $\Omega_{I}=a+(b-a) \Omega$; we see immediately that $\Omega_{I}$ has the following properties:

(1) $\Omega_{I}$ is in the interior of $I$,

(2) $\Omega_{I}[s]:=a+(b-a) \Omega_{s}$ is a monotone approximation to $\Omega_{I}$, with range contained in $I$,

(3) $\Omega_{I}$ is Martin-Löf random, and

(4) $\Omega_{I}$ is not difference random. 
We define a procedure $\mathcal{M}(I, E, \epsilon)$ that works for a requirement $\mathcal{R}_{E}$ inside the interval $I$ and adds measure at most $\epsilon 2^{-n}$ to the $n$th level of the test we build. We need to succeed at requirement $\mathcal{R}_{E}$ inside the interval $I_{E}$; the reason we define things in terms of procedures $\mathcal{M}(I, E, \epsilon)$ is that if $E=E^{\prime} \cup\{e\}$ and we succeed at requirement $\mathcal{R}_{E^{\prime}}$ inside an interval $I$ in the complement of $\mathscr{D}_{e}$, then we also succeed at requirement $\mathcal{R}_{E}$. Therefore, our strategy for $\mathcal{R}_{E}$ involves finding such a pair $e, E^{\prime}$ and interval $I$ and recursing to perform the strategy for $\mathcal{R}_{E^{\prime}}$ inside that interval.

The definition of the procedure $\mathcal{M}(I, E, \epsilon)$ depends on the stage $s_{0}$ when it is called and on whether the set $E$ is empty. If $E$ is nonempty, then it looks for a place to recurse and call a subprocedure for $E \backslash\{e\}$, but if $E$ is the empty set, then it merely has to ensure that $\mathcal{T}^{A}$ captures some element of the interval $I$.

3.1 Procedure $\mathcal{M}(\boldsymbol{I}, \boldsymbol{E}, \boldsymbol{\epsilon})$, for $\boldsymbol{E}$ nonempty Stage $s_{0}$ : We assume that $\mathcal{U}_{n}^{A}\left[s_{0}\right] \cap$ $I=\varnothing$ for all $n$ and that $\bigodot^{A}\left[s_{0}\right] \cap I=I$. Procedure $\mathcal{M}(I, E, \epsilon)$ has two states, "searching" and "found," and begins in the "searching" state.

Stage $s>s_{0}$ : We have the following.

- Step 1: If this procedure is in the "found" state, then go to step (2) below. If this procedure is in the "searching" state, then search for the least pair $(a, b)$ of rationals according to the lexicographic order on $\mathbb{Q}^{2}$ such that

(1) $\Omega_{I}[s-1]<a<b<\Omega_{I}[f(s)]$,

(2) there is some $e \in E$ such that the open interval $(a, b)$ is a subset of the complement of $\mathscr{D}_{e}[f(s)]$, and

(3) the denominators of $a$ and $b$ are at most $f(s)$.

If such a pair is found, then switch to the "found" state and begin the procedure $\mathcal{M}((a, b), E \backslash\{e\}, \epsilon / 2)$.

- Step 2: If this procedure is in the "searching" state, then let $\hat{I}=I$; if this procedure is in the "found" state and $(a, b)$ is the interval it found, then let $\hat{I}=I \backslash[a, b]$. Enumerate $\hat{I} \cap\left[0, \Omega_{I}[s]+\epsilon 2^{-n-1}\right)$ into $U_{n}^{A}$ for each $n$, and enumerate $\hat{I} \cap\left[0, \Omega_{I}[s]\right)$ into the complement of $\mathcal{C}^{A}$.

3.2 Procedure $\mathcal{M}(I, E, \epsilon)$, for $\boldsymbol{E}$ empty Stage $s_{0}$ : We assume that $\mathcal{U}_{n}^{A}\left[s_{0}\right] \cap I=\emptyset$ for all $n$, and that $\ell^{A}\left[s_{0}\right] \cap I=I$. Let $q$ be the midpoint of the interval $I$.

Stage $s_{0}+1$ : Enumerate $I$ into $\mathcal{U}_{n}$, and enumerate $I \backslash\{q\}$ into the complement of $\mathcal{C}^{A}$.

No action will be taken by this procedure at stages after $s_{0}+1$.

3.3 Construction Let $E_{s}$ denote the finite set of natural numbers with canonical index $s$.

At stage $s$, call the procedure $\mathcal{M}\left(I_{E_{s}}, E_{s}, \epsilon_{E_{S}}\right)$, and take one step in each procedure already called.

3.4 Verification When one procedure $\mathcal{M}(I, E, \epsilon)$ calls another procedure $\mathcal{M}\left(I^{\prime}\right.$, $\left.E^{\prime}, \epsilon^{\prime}\right)$, call the latter procedure a subprocedure of the former. We inductively define $\mathcal{M}\left(I^{\prime}, E^{\prime}, \epsilon^{\prime}\right)$ to be a child of $\mathcal{M}(I, E, \epsilon)$ (and the latter a parent of the former) if $\mathcal{M}\left(I^{\prime}, E^{\prime}, \epsilon^{\prime}\right)$ is a subprocedure of $\mathcal{M}(I, E, \epsilon)$ or a child of a subprocedure of $\mathcal{M}(I, E, \epsilon)$. Looking at the procedure and construction definitions, one can verify the following properties:

- Each procedure has at most one subprocedure. 
- No action taken by $\mathcal{M}(I, E, \epsilon)$ can affect any part of $U_{n}^{A}$ or $\mathcal{C}^{A}$ outside of $I$.

- If $\mathcal{M}(I, E, \epsilon)$ calls $\mathcal{M}\left(I^{\prime}, E^{\prime}, \epsilon^{\prime}\right)$ at a stage $s$, then $I^{\prime}$ is a proper subinterval of $I$, and the procedure $\mathcal{M}(I, E, \epsilon)$ has not affected any part of $U_{n}^{A}$ or $\ell^{A}$ inside $I^{\prime}$ before the end of stage $s$ and will never subsequently do so.

- The only procedures called by the construction are the procedures $\mathcal{M}\left(I_{E}\right.$, $\left.E, \epsilon_{E}\right)$ and their children.

By the last two properties, if two procedures $\mathcal{M}(I, E, \epsilon)$ and $\mathcal{M}\left(I^{\prime}, E^{\prime}, \epsilon^{\prime}\right)$ are called during the construction, then either $I$ and $I^{\prime}$ are disjoint or one is a proper subinterval of the other and the corresponding procedure is a child of the other procedure. For each procedure $\mathcal{M}(I, E, \epsilon)$ called during the construction, define $\hat{I}$ to be $I \backslash I^{\prime}$, where $\mathcal{M}\left(I^{\prime}, E^{\prime}, \epsilon^{\prime}\right)$ is the unique subprocedure of $\mathcal{M}(I, E, \epsilon)$ (or $\hat{I}=I$ if $\mathcal{M}(I, E, \epsilon)$ has no subprocedure). Since at most one procedure is called with interval $I$ as the first argument and each procedure calls at most one subprocedure, this is well defined. Finally, the four properties together imply that the action of procedure $\mathcal{M}(I, E, \epsilon)$ is the only thing that can affect any part of $\mathcal{U}_{n}^{A}$ or $\mathcal{C}^{A}$ inside $\hat{I}$ during the construction.

If $E$ is nonempty, then the procedure $\mathcal{M}(I, E, \epsilon)$ enumerates all of the interval $I \cap\left[0, \Omega_{I}+\epsilon 2^{-n-1}\right)$ into $U_{n}^{A}$, except for the interval $I^{\prime}$ if a subprocedure $\mathcal{M}\left(I^{\prime}, E^{\prime}, \epsilon^{\prime}\right)$ is called. Therefore,

$$
\hat{I} \cap U_{n}^{A}=\hat{I} \cap\left[0, \Omega_{I}+\epsilon 2^{-n-1}\right) .
$$

If $E$ is nonempty, then $\mathcal{M}(I, E, \epsilon)$ also enumerates all of the interval $I \cap\left[0, \Omega_{I}\right)$ into the complement of $\mathcal{C}^{A}$, excluding the interval $I^{\prime}$ if a subprocedure $\mathcal{M}\left(I^{\prime}, E^{\prime}, \epsilon^{\prime}\right)$ is called. Hence if a procedure $\mathcal{M}(I, E, \epsilon)$ is called during the construction with $E$ nonempty, then $\hat{I} \cap \mathcal{C}^{A}=\hat{I} \cap\left[0, \Omega_{I}\right)$. If $E$ is empty, then $\hat{I}=I, I \subset U_{n}^{A}$ for all $n$, and $I \cap \mathcal{C}^{A}=\{q\}$, where $q$ is the midpoint of $I$.

Lemma 15 If the procedure $\mathcal{M}(I, E, \epsilon)$ is called during the construction, then

$$
\mu\left(I \cap U_{n}^{A} \cap \mathcal{C}^{A}\right) \leq \epsilon 2^{-n} .
$$

Proof We prove the lemma by induction on the size of $E$. If $E$ is empty, then by the above we have $I=\hat{I}$ and $I \cap \mathcal{U}_{n}^{A} \cap \mathcal{C}^{A}$ is the singleton $\{q\}$, where $q$ is the midpoint of $I$, and hence $I$ has measure 0 . If $E$ is nonempty and no subprocedure is called by $\mathcal{M}(I, E, \epsilon)$, then we again have $I=\hat{I}$, and

$$
I \cap \mathcal{U}_{n}^{A} \cap \mathcal{C}^{A}=I \cap\left[\Omega_{I}, \Omega_{I}+\epsilon 2^{-n-1}\right),
$$

so the measure is at most $\epsilon 2^{-n-1}$. If $E$ is nonempty and $\mathcal{M}(I, E, \epsilon)$ calls a subprocedure $\mathcal{M}\left(I^{\prime}, E^{\prime}, \epsilon^{\prime}\right)$, then $\left|E^{\prime}\right|=|E|-1$ and $\epsilon^{\prime}=\epsilon / 2$, so by the inductive hypothesis,

$$
\mu\left(I^{\prime} \cap \mathcal{U}_{n}^{A} \cap \bigodot^{A}\right) \leq \epsilon 2^{-n-1} .
$$

Also,

$$
\hat{I} \cap \mathcal{U}_{n}^{A} \cap \mathcal{C}^{A}=\hat{I} \cap\left[\Omega_{I}, \Omega_{I}+\epsilon 2^{-n-1}\right),
$$

which has measure at most $\epsilon 2^{-n-1}$. Hence

$$
I \cap U_{n}^{A} \cap \mathcal{C}^{A}=\left(\hat{I} \cap u_{n}^{A} \cap \mathcal{C}^{A}\right) \cup\left(I^{\prime} \cap U_{n}^{A} \cap \bigodot^{A}\right)
$$

has measure at most $\epsilon 2^{-n}$.

Thus

$$
\mu\left(I_{E} \cap u_{n}^{A} \cap \bigodot^{A}\right) \leq 2^{-n} \epsilon_{E}
$$


and

$$
\mu\left(U_{n}^{A} \cap \mathcal{C}^{A}\right)=\mu\left(\bigcup_{E} I_{E} \cap u_{n}^{A} \cap \mathcal{C}^{A}\right) \leq \sum_{E} 2^{-n} \epsilon_{E}=2^{-n},
$$

so $\left(\left\langle U_{n}^{A}\right\rangle, \bigodot^{A}\right)$ is a difference test relative to $A$.

Lemma 16 The A-difference test $\mathcal{T}^{A}=\left(\left\langle u_{n}^{A}\right\rangle, \mathcal{C}^{A}\right)$ is not covered by any finite ensemble of unrelativized difference tests.

Proof We say that $\mathcal{R}_{E}$ is satisfied inside $I$ if there is some real $X \in I$ which is captured by the test $\mathcal{T}^{A}$ but is not captured by any of the tests $\mathcal{T}_{e}$ for $e \in E$. (In the case when $E$ is empty, this just says that $\mathcal{T}^{A}$ captures some real in the interval $I$.) We will show that for every set $E$, the requirement $\mathcal{R}_{E}$ is satisfied inside $I_{E}$. Notice that if $e \in E$ and $E^{\prime}=E \backslash\{e\}$, then if $\mathcal{R}_{E^{\prime}}$ is satisfied on some interval $I^{\prime} \subset I$ in the complement of $\mathscr{D}_{e}$, there is some real $X \in I^{\prime}$ not captured by any of the tests $\mathcal{T}_{e^{\prime}}$ for $e^{\prime} \in E^{\prime}$. Since $X \notin \mathscr{D}_{e}$, it is also not captured by $\mathcal{T}_{e}$, and hence $\mathcal{R}_{E}$ is satisfied inside $I$.

We will prove by induction on $|E|$ that if a procedure $\mathcal{M}(I, E, \epsilon)$ is called during the construction, then $\mathcal{R}_{E}$ is satisfied inside $I$.

Suppose that $E$ is empty. We showed above that in this case, $\mathcal{T}^{A}$ captures the midpoint of $I$. Hence $\mathcal{R}_{E}$ is satisfied inside $I$.

Now suppose that $E$ is nonempty. We showed above that $\Omega_{I} \in I$ and $\Omega_{I}$ is captured by the $A$-difference test $\mathcal{T}^{A}$, so if $\Omega_{I}$ is not also captured by one of the difference tests $\mathcal{T}_{e}$ where $e \in E$, then $\mathcal{R}_{E}$ is satisfied inside $I$. Suppose that $\Omega_{I}$ is captured by at least one of these tests. If the procedure $\mathcal{M}(I, E, \epsilon)$ eventually goes from the "searching" state to the "found" state after finding some subinterval $I^{\prime}=(a, b)$ in the complement of some $\mathscr{D}_{e}$ (with $e \in E$ ) and calling the subprocedure $\mathcal{M}\left(I^{\prime}, E^{\prime}, \epsilon / 2\right)$ (where $E^{\prime}=E \backslash\{e\}$ ), then $\left|E^{\prime}\right|=|E|-1$, so by the inductive hypothesis we may assume that $\mathcal{R}_{E^{\prime}}$ is satisfied inside $I^{\prime}$. But as shown above, this implies that $\mathcal{R}_{E}$ is satisfied inside $I$. So it suffices to show that if $\Omega_{I}$ is captured by at least one of the tests $\mathcal{T}_{e}$ where $e \in E$, then the procedure $\mathcal{M}(I, E, \epsilon)$ eventually goes from the "searching" state to the "found" state.

Let $s_{0}$ be the stage at which the procedure $\mathcal{M}(I, E, \epsilon)$ is called. Fix $e \in E$ such that $\mathcal{T}_{e}$ captures $\Omega_{I}$. As before, in any interval of the form $\left(\Omega_{I}[s-1], \Omega_{I}\right)$, there must be some rational subinterval removed from $\mathscr{D}_{e}$. We will define a computable function $g: \omega \rightarrow \omega$. To define $g(s)$, search for some natural number $n$ and pair of rational numbers $a, b$ with denominator at most $n$, such that $\Omega_{I}[s-1]<a<b<\Omega_{I}[n]$ and the interval $(a, b)$ is in the complement of $\mathscr{D}_{e}[n]$; let $g(s)$ be the least such $n$. Such an $n$ will always be found, and so $g$ is a total computable function. Since $f$ escapes domination by $g$, there is a stage $s>s_{0}$ with $f(s)>g(s)$. During stage $s$ of the construction, the search for a rational interval $(a, b)$ meeting conditions (1)-(3) must succeed, so the procedure $\mathcal{M}(I, E, \epsilon)$ will move to the "found" state at stage $s$, provided that it is still searching.

This completes the proof.

\section{Note}

1. In the space $[0,1]$, intervals of the form $[0, x)$ are indeed open. 


\section{References}

[1] Bienvenu, L., and J. S. Miller, "Randomness and lowness notions via open covers," Annals of Pure and Applied Logic, vol. 163 (2012), pp. 506-18. Zbl 1250.03067. MR 2880269. DOI 10.1016/j.apal.2011.06.009.

[2] Day, A. R., and J. S. Miller, "Cupping with random sets," to appear in Proceedings of the American Mathematical Society preprint, arXiv:1206.1603v1 [math.LO].

[3] Downey, R. G., and D. R. Hirschfeldt, Algorithmic Randomness and Complexity, Springer, New York, 2010. MR 2732288. DOI 10.1007/978-0-387-68441-3. 63

[4] Downey, R. G., A. Nies, R. Weber, and L. Yu, "Lowness and $\Pi_{2}^{0}$ nullsets," Journal of Symbolic Logic, vol. 71 (2006), pp. 1044-52. MR 2251554. DOI 10.2178/js1 /1154698590. 67

[5] Franklin, J. N. Y., "Lowness and highness properties for randomness notions," pp. 124-51 in 10th Asian Logic Conference, World Scientific, Hackensack, N.J., 2010. MR 2798897. DOI 10.1142/9789814293020_0006. 63

[6] Franklin, J. N. Y., and K. M. Ng, "Difference randomness," Proceedings of the American Mathematical Society, vol. 139 (2011), pp. 345-60. Zbl 1214.03029. MR 2729096. DOI 10.1090/S0002-9939-2010-10513-0. 64, 67

[7] Franklin, J. N. Y., and R. Solomon, "Degrees that are low for isomorphism," in preparation. 63

[8] Kjos-Hanssen, B., A. Nies, and F. Stephan, "Lowness for the class of Schnorr random reals," SIAM Journal on Computing, vol. 35 (2005), pp. 647-57. Zbl 1095.68043. MR 2201451. DOI 10.1137/S0097539704446323. 64

[9] Nies, A., "Lowness properties and randomness," Advances in Mathematics, vol. 197 (2005), pp. 274-305. Zbl 1141.03017. MR 2166184. DOI 10.1016/j.aim.2004.10.006. 67

[10] Nies, A., Computability and Randomness, vol. 51 of Oxford Logic Guides, Oxford University Press, Oxford, 2009. MR 2548883. DOI 10.1093/acprof:oso/9780199230761 .001.0001. 63, 64

[11] Slaman, T. A., and R. M. Solovay, "When oracles do not help," pp. 379-83 in Fourth Annual Workshop on Computational Learning Theory (Santa Cruz, CA, 1991), Morgan Kaufman, San Francisco, 1991.

[12] Stephan, F., "Martin-Löf Random and PA-complete Sets," Technical Report 58, Matematisches Institut, Universität Heidelberg, Heidelberg, 2002.

[13] Terwijn, S. A., and D. Zambella, "Computational randomness and lowness," Journal of Symbolic Logic, vol. 66 (2001), pp. 1199-1205. Zbl 0990.03033. MR 1856736. DOI 10.2307/2695101. 64

\section{Acknowledgments}

This work was done at the Dagstuhl seminar on Computability, Complexity, and Randomness in January 2012. The authors thank the organizers of the seminar and the staff at Dagstuhl for providing such a pleasant and productive working environment.

\section{Diamondstone}

Department of Mathematics, Statistics, and Computer Science

Victoria University of Wellington

PO Box 600

Wellington 6140

New Zealand

ddiamondstone@gmail.com 
Franklin

Department of Mathematics

196 Auditorium Road

University of Connecticut, Unit 3009

Storrs, Connecticut 06269-3009

USA

johanna.franklin@uconn.edu 